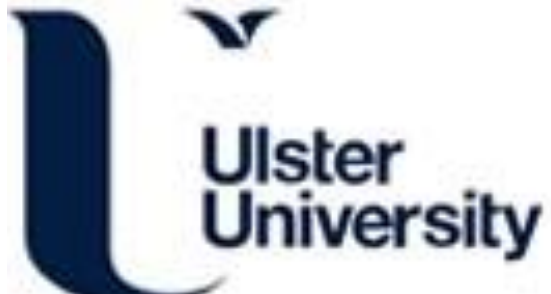

Hospital-based social work: Challenges at the interface between health and social care

Heenan, D., \& Birrell, D. (2019). Hospital-based social work: Challenges at the interface between health and social care. The British Journal of Social Work, 49(7), 1741-1758. https://doi.org/10.1093/bjsw/bcy114

Link to publication record in Ulster University Research Portal

\section{Published in:}

The British Journal of Social Work

Publication Status:

Published (in print/issue): 31/10/2019

DOI:

10.1093/bjsw/bcy114

Document Version

Author Accepted version

\section{General rights}

Copyright for the publications made accessible via Ulster University's Research Portal is retained by the author(s) and / or other copyright owners and it is a condition of accessing these publications that users recognise and abide by the legal requirements associated with these rights.

\section{Take down policy}

The Research Portal is Ulster University's institutional repository that provides access to Ulster's research outputs. Every effort has been made to ensure that content in the Research Portal does not infringe any person's rights, or applicable UK laws. If you discover content in the Research Portal that you believe breaches copyright or violates any law, please contact pure-support@ulster.ac.uk. 


\title{
Hospital-based social work: Challenges at the interface between health and social care.
}

\begin{abstract}
This article analyses the complexity and challenges of hospital-based social work in the rapidly changing and financially driven climate of health and social care. It is based on the findings of an exploratory qualitative study of social work in an acute hospital in Northern Ireland. It considers the intensity and variety of social work practice in this clinical setting and explores the challenges and rewards of interprofessional collaboration. It suggests that hospital-based social workers occupy a unique place at the interface of health and social care, yet relatively little is known about their role and contribution. Whilst these highly skilled professionals play a pivotal part in ensuring the wellbeing of service users, their traditional roles have become secondary to co-ordinating hospital discharge. It is argued that the focus on avoiding delayed discharges and increasing patient throughput may overshadow their strategic and value based contributions. Finally, it is clear that inter-professional working can improve health outcomes and facilitate optimum wellbeing, this study highlights a number of areas of concern for social workers working in these teams.
\end{abstract}

\section{Introduction}

Hospital based social work practice has been in existence in the United Kingdom since the late $19^{\text {th }}$ century, when social care professionals were required to assess whether or not people were deserving of treatment. Despite this long historical tradition and the apparent value of their services, their contributions have received 
scant attention (Beder, 2006, Barber et al, 2015, Cleak, 2014, McLaughlin, 2016).

Hospital social workers have multi-dimensional roles across a range of domains including education, advocacy and counselling providing crucial support to those who are often frail and vulnerable (Bendor, 1998, Craig and Muskat, 2013). They assist service users and their families understand and come to terms with the impact of illness and treatments and deliver a range of interventions that aim to ensure that the individual is receiving the most appropriate care. Significantly, they take a holistic approach to a person's care needs which involves understanding the wishes of the service user and their families. Often this entails balancing complex family dynamics, advocating in the best interests of the service user to achieve a safe and efficient discharge from the hospital. Health based social work operates beyond the immediate medical concerns of patients and into the broader ecological context and its impact on health outcomes (Cleak, 2014). In the last decade, discharge planning has emerged as a major professional function in hospital settings and represents an integral part of practice. In the field of healthcare discharge planning is usually considered as the core function of the hospital-based social worker. Inefficient and inappropriate hospital discharges are a major policy issue for the design and delivery of health services, yet the contribution of the hospital social worker to this process remains poorly understood. This paper assesses the main roles of hospital-based social workers and identifies the key challenges they face working in inter-disciplinary health care teams. It concludes that the emphasis on discharge planning and targets has impacted on other more traditional aspects of their role and working in an inter-disciplinary context may have implications for models of social work education. 


\section{Background}

For over a century in the UK, hospital-based social work has played a crucial role in delivering a person-centred response to the needs of sick and vulnerable people at a time of unexpected difficulties. By engaging with patients and their families they can address the social and emotional impacts of hospital admission. They work collaboratively to assist individuals and their families deal with challenges and find the solutions that enable them to live safely and independently within their communities. Their unique knowledge, skills and values are applied to improve people's resilience and help them to realise their full potential and ensure wellbeing. Cowles and Lefcowitz (1992) describe their approach as bio-psychosocial, as it considers an individual within the context of his or her wider environment. Crucially, they ensure that the service users and their families are central to any decision-making process. They assist individuals to navigate the unfamiliar clinical context and address social and emotional needs. Drawing on their knowledge of the services and assets available in the wider community, they aim to maximise the impact of limited resources. Historically, discharge planning has been considered a key feature of their role (Holliman et al 2001, McAlynn and McLaughlin, 2008, McLaughlin, 2016). In the last decade however this aspect of their work has taken on increasing significance and is widely viewed as their central function (Davis et al , 2005, Auberbath et al, 2007, Fantus et al 2017). In their US study, Judd and Sheffield, (2010) reported that approximately $60 \%$ of social work time in hospitals was spent on discharge planning, including securing and coordinating support services and organising alternative longterm care for patients. Hospital discharge is not a single or isolated event but is rather a complex series of linked incremental steps that involves a wide range of groups and networks sharing knowledge and making decisions. 
Against a backdrop of austerity and increasing demand for healthcare reducing delayed discharge from hospitals has risen up the policy agenda in the UK (Glasby, 2004, Glasby, 2007, Majeed et al, 2012, NHS Providers, 2015). The rationale for this concern is that a considerable body of evidence suggests that hospital discharge is a complex issue characterised by poor patient experience, fragmentation, duplication, inadequate communication, insufficient collaboration, delay and inefficiencies. Whilst this is the case across all user groups, it is particularly true for older people who are major users of health and social services (Abendstern et al 2011, Cornwell et al 2012, NAO, 2016). Longer stays in hospital have a disproportionately negative effective on older people as they quickly lose their strength and mobility (Oliver et al, 2014, Oliver, 2015). As well as the human cost, this represents poor value for money and places avoidable financial pressures on the NHS. Despite the fact that discharge planning is viewed as a critical function in health care, recent reports suggest that the number of delayed discharges has been increasing at an 'alarming rate' with 'far too many' people in hospitals who do not need to be there. It was been suggested that without 'radical action' the problem will worsen and the system will become unsustainable (NIAO, 2016, NHS Providers 2015, NHS England 2017). There is a recognition of the need for a deeper, evidence based understanding of the complex organisational and inter-professional issues which underpin this issue (Swinkels, et al, 2009).

There is a general agreement in the national and international literature that effective hospital discharge planning must be underpinned by inter-disciplinary team working (Pethybridge, 2004, Lin and Tjung, 2012, Barber, 2015, Ambrose-Millar and 
Ashcroft, 2016). Developing and sustaining successful inter-professional relationships facilitate optimal health outcomes. Inter-disciplinary teams help to break down barriers between professional groups and foster a sense of common purpose and trust (Bull and Roberts, 2001). In the UK social workers are usually identified as the lead professionals in discharge planning. As such they are charged with supporting the integration of healthcare professionals, overseeing the planning process and addressing emergent issues in a responsive manner. They take the lead in terms of coordinating the process, mitigating the complexity of the system and facilitating knowledge sharing and are responsible for both in-reach within the system and outreach to other services. Importantly, they are the primary point of contact for the service user working with them from admission to discharge and often continuing through to community care. Although it is generally accepted that social workers have the ability to bring a unique perspective to inter-disciplinary teams, research suggest the dominance of the medical model in this clinical setting can create a number of challenges (Beddoe, 2013, Oliver 2013, Ambrose-Millar and Ashcroft 2016). Social workers working in health care must be professionally assertive in order to protect their values, identity and expertise (Beddoe, 2013). Ambrose-Miller and Ashcroft (2016) note that inter-disciplinary collaboration in healthcare presents both challenges and rewards for social workers. Culture, power dynamics, poor communication and a lack of clarity around roles can hamper effective collaboration.

In his study of the relationship between health and social care professionals in England (Hudson, 2002) noted that the two main professional groupings charged with delivering interdisciplinary care were social workers and nurses. The relationship between them was described as 'far from comfortable'. The tension was explained by 
disagreement over prioritisation, competition over resources and antagonism over blurred role boundaries. Research from Saudi Arabia (Albrithen and Yalli, 2016) suggested that the effectiveness of social workers in inter-disciplinary teams was constrained by the dominance of the medical professionals, power dynamics, ineffective communication and a lack of clarity around roles. Glasby (2004) stressed that hospital social workers were highly valued by their colleagues, but in an underpressure healthcare system tensions could quickly emerge between social workers and other professionals when the time needed to make psychosocial assessments caused delays to the discharge process. Intensive discharge pressures and a managerial focus on targets and efficiencies can create a difficult environment for social workers undertaking holistic assessments of vulnerable people and their families.

Oliver (2013) cautioned that professional social work identity could be blurred by the integrative nature of inter-professional teams where competence rather than professional scope determines how each member will contribute. This mode of working typically requires doing things differently from the professional norm. $\mathrm{He}$ distinguishes this from multi-professional work where different specialities collaborate but traditional lines of authority, power relationships and professional knowledge boundaries are maintained. In inter-professional teams individuals must adapt sufficiently to each other to produce shared objectives and a joint vision. Consequently, inter-professional work can lead to confusion around roles, unclear group boundaries and the relinquishing of expert status, which may be considered a serious threat to professional identity. 
Some of the dilemmas and difficulties experienced by social workers working in the healthcare setting relate to retaining their own values, ethics and professional identity. They may experience ethical conflicts around employers' codes and guidance and their professional code of ethics (Banks, 2004). Social workers are also acutely aware of the consequences of austerity and how spending cuts impacted on their ability to deliver services and interventions. Employers want workers who are committed to maximising scarce resources, rather than ones concerned with empowerment and social justice. Implementing strategies and protocols aimed at rationing resources and managing budgets can lead to ethical challenges for the practice of social work.

\section{Methodology}

This paper was based on a small-scale qualitative study undertaken in an acute hospital in the Western Health and Social Care Trust, one of Northern Ireland's five integrated health and social care trusts. It has responsibility for 300,000 people, which represents approximately $16 \%$ of the total population. The study was funded by the Western Health and Social Care Trust and was completed in a six-month period. As the research was exploratory, a qualitative approach was adopted. Following an initial assessment of social work in this clinical setting, it was decided to purposively sample the social work teams working with older people. In line with other regions of the UK, society in Northern Ireland is getting older: people are living longer, often with multiple, long-term health conditions, and are having fewer children. It has been estimated that by 2026 , for the first time, there will be more over 65 s than under $16 \mathrm{~s}$. By 2039, the population aged 65 and over will have increased by $74 \%$ compared to the position in 2014. Consequently, one in four people will be aged 65 and over. Similarly, the population aged 85 and over will increase by $157 \%$ over the same 
period, which will see their share of the population increase from $1.9 \%$ to $4.4 \%$

(DHSSPS, 2016). Whilst living longer is a cause for celebration, it also represents a huge and growing challenge in terms of the demands and pressures on health and social care services.

The study involved semi-structured, face-to face interviews with 33 health and social care staff including 13 hospital-based and 6 community social workers, 3 members of social work management, 5 nurses, 2 occupational therapists, 2 physiotherapists, 2 general practitioners, a consultant in geriatric medicine. This was a relatively small team of information-rich respondents who were articulate and willing to reflect on working in this context. In this hospital, social workers assumed the lead responsibility for discharge planning. The staff were based in wards dealing primarily with older people. The research conforms to internationally accepted ethical guidelines approved by the relevant university committee and all participants gave their verbal consent. The interviews aimed to provide perspectives of various professionals on the role of the social worker, with particular reference to their contribution to the hospital discharge process. A short interview guide with five openended questions was used to facilitate greater consistency in the data and enable participants discuss their experiences and perceptions. The methodology included a mix of one to one interviews and small group interviews. The social workers and hospital-based professionals were interviewed in focus groups. The director of social work and the social work manager were interviewed on a one to one basis as they were concerned that their presence could impact on participants willingness to speak freely. The GPs were also interviewed on a one to one basis for logistical reasons as they are not based on the hospital site. Each interviewee was assigned a reference number 1-33 and this number is included at the end of direct quotes. These interviews 
were recorded and transcribed and analysed using thematic analysis to identify meaning and concepts from the data and pinpointing themes. Themes were developed from the data by the two researchers independently coding the transcripts and then comparing notes to improve rigour. The research also involved finding relationships between the categories and accounting for these relationships. Thematic analysis is commonly used in health research to build theory, as its inductive nature can help to explain experiences and perceptions (Javadi and Zarea, 2016). The findings are presented under five key headings the role of hospital-based social workers, challenges at the health and social care interface, interdisciplinary working, ethical issues and improving service provision.

\section{Guide to responses}

Hospital-based social workers 1-13

Community-based social workers 14-19

Social work management 20-22

Nurses 23-25

Physiotherapists and Occupational Therapists 26-29

General Practitioners 30-32

Consultant 33

\section{The role of hospital-based social workers.}

The role of social workers working in an acute hospital in an integrated health and social care system can be broadly defined as assessing the health and social care needs of the service-user and their families and ensuring their social wellbeing. The 
social workers in this study described their roles as difficult to define as they were multi-dimensional, wide and varied. They managed complex family dynamics, usually at a point of crisis whilst working to achieve the best outcomes for their clients. It was described as a balancing act, an attempt to achieve the best outcomes for all concerned. Acting as an advocate for service users and their families was identified as one of the their key functions. Respondents stressed that many of the older patients were frightened by the decision-making process and were frequently surrounded by people giving conflicting information and advice. In this context it was essential to have someone taking a comprehensive approach to need and ensuring that service users were fully informed and empowered to make decisions.

For me, our job is all about acting in the interests of the patients and their families, advocating for them. Everything else I do is seen through that lens. Often when people are admitted we are seeing them at their worst, we reassure, comfort and give some peace of mind. \#3

We advocate and empower that's how I would describe it. It is our role to ensure that people are not passive and powerless in decisions that really affect their lives. We ensure that they have a voice and that it is heard. \#5

I think our advocacy role is the crucial aspect of what we do. We have the knowledge and skills to act on behalf of the service user. \#6

There was also general agreement that within this clinical environment their focus on a holistic assessment of need was also a key part of their role. This required particular knowledge, skills, values and expertise to inform the decision making process. This comprehensive approach was viewed as the basis of initial and ongoing patient care. Beder (2006) suggested that the hospital social worker brings a specific biopsychosocial approach that takes account of the needs of the whole person and their 
environment and this informs interventions. The holistic aspect of their role was commented on by many of the respondents.

We are not just interested in the illness we are concerned with the much broader dimensions of care, the family, the community, the resources than can be drawn on support and enhance quality of life. We negotiate, we arrange, we plead, we beg, we insist, all to get the best results. Sometimes we seem like the odd man out. \#10

Our big picture approach is what separates us from the others working in the hospital. If someone is medically fit, but their circumstances mean they can't go home then its over to the social workers. \#13

Social workers see the whole person not just the illness. They don't just look at a hip or a knee but the person and their family and their circumstances. Also they can read between the lines and form an assessment of what is best for the older person. \#14

Dealing with emotional distress and being empathic were also identified as central aspects of this work. Empathy has been identified as particularly important to social work and clients as it is associated with improved outcomes (Gerdes and Segal 2011). In this study it equated to showing concern, building rapport and trust ensuring that older patients felt safe and cared for. One respondent explained it as follows, Sometimes you can get frustrated as you always seem to be firefighting and it can be difficult to establish trust with people when they most need your help. But it is crucial to provide emotional support, to listen, to understand. Connecting with others is essential. \#7

There was a general agreement that coordinating timely hospital discharge plans was a pivotal aspect of the social work role. This involved the development and implementation of a safe discharge plan that ensures the wellbeing of the patient. Whilst this was an inter-disciplinary activity, social workers were the designated professionals who assumed the primary responsibility for coordinating this service. 
They navigate and respond to the competing pressures in this clinical setting.

According to Oktay et al (1992) their comprehensive knowledge of complex systems of health and social care ensure services are appropriately allocated. One of the nurses suggested that social workers seemed ideally placed to address fragmentation and ensure that individuals experienced a seamless service.

In many ways social workers are the only ones who are equipped to undertake this role. They have the knowledge and the skills to put together a plan that will work for the patient and their family. It's the breath of knowledge and their ability to negotiate the best outcomes. \#23

The complexity of this work and the fact that there was no template or no one size fits all approach was stressed by the social workers. This task required a range of skills and knowledge and was not simply a matter of 'seeing to the paperwork'.

No two discharge plans are the same. Most patients have complex needs that cannot be easily assessed, it's not like a quick check-up. We have to balance risk, and ensure that there is continuity of care when someone leaves the hospital. \#8

\section{Challenges at the health and social care interface}

From the interviews, it emerged that whilst this hospital-based role was rewarding, it also presented a number of challenges and tensions, particularly around discharge processs and inter-disciplinary working. There was a broad agreement that developing and implementing discharge plans had become the overwhelming focus of hospital social work and significantly that this had negatively impacted on other more traditional aspects of their work. As McLaughlin, (2016) suggested intensive discharge pressures and a managerial focus on targets and measurable outputs can 
jeopardize holistic assessments and the needs of vulnerable patients. A social worker explained it as follows,

Of course our role in hospital discharge is important, but it is almost as though we have become one trick ponies. You don't have time to build relationships or give emotional support with people and gain their trust as we are far too busy chasing up some assessment or other. \#3

In this clinical environment there was tension between discharge management and what could be described as a more traditional therapeutic role.

Sometimes with the pressure to get people moving as quickly as possible there is a temptation to simply accept the views of other professionals but for us it is crucial to advocate for the service user. We must never lose sight of that. \#16

Whilst there was an acceptance that the social work role in discharge planning was vital, there was also some frustration at the amount of time spent on the associated bureaucratic and administrative tasks. It was suggested that this was a poor use of time and resources and the time spent on paperwork negatively impacted on the perception of their role.

We just don't have time to spend with families and provide the level of support that we would like to as now it is all about procedures, strategies and protocols. We have to constantly push back on this. \#20

In a busy hospital sometimes it seems like discharge planning is the only show in town, it can be easy to lose focus on our role. \#22

It can be bloody frustrating to always be seen as the person keeping someone in hospital but we have to focus on our job and that's keeping people safe. \#7

\section{Inter-disciplinary working}


Overall inter-disciplinary working was described as successful and facilitated by the organisational structure of an integrated system of health and social care (Heenan and Birrell, 2009, Heenan and Birrell, 2018). There was a recognition from the health care professionals that social workers brought a unique perspective to the team. In this hospital inter-disciplinary teams shared the same goals of ensuring the best outcomes for those using the health care system. It was suggested that there was a collaborative culture in this organisation that supported and valued working together. A physiotherapist described the work of this team as 'a group of like minded people working together to achieve shared outcomes'. There was a strong consensus that the co-location of the health and social care workers in the building was essential to build relationships and establish an informed understanding of the team dynamics.

A consultant described social workers as bridges,

I mean its health and social care so it makes perfect sense to have social workers in the hospital. They build relationships with the staff, but also have a wealth of knowledge and understanding about what is going on in the community. They are a bridge and one that we couldn't do without. \#33

This view was reiterated by a nurse who suggested inter-disciplinary work facilitated collaboration and mutual respect.

An important part of our job is working together as a team, all pulling in the same direction. It is just common sense when you think about it. To be honest with you before I had this job I hadn't a clue what social worker's did, I actually didn't know that they worked in the hospital. \#25

Whilst social workers were described as invaluable and brought a different perspective to the team, both the hospital and community based social workers 
expressed some concern regarding a perceived lack of clarity about their professional identity. On the one hand they were described by healthcare colleagues as "the lynchpin' or "the glue"of the inter-disciplinary team, there was a strong concern that their skills and expertise were poorly understood. Their ability to get things done and access resources was acknowledged and valued, however their professional philosophy had caused tensions. Some participants highlighted the need to ensure clarity around the social work contribution and identified a lack of knowledge as one of the factors hindering their capacity to meet the needs of service users.

In many ways we seen just the dogs bodies or glorified paper pushers. Our actual contribution and the skills we bring to the table are really not well understood and acknowledged. Sometimes its frustrating wasting time explaining when we should just be getting on with it. \#15

Everybody knows what a nurse does or a doctor does, most people know about physiotherapists, we are the people who ask awkward questions and are best avoided. \#12

You have to earn respect when working here. No point in saying I'm ignored if you have nothing to contribute. We have to demonstrate our value and worth our knowledge and skills and then people will listen. I think the idea that the medics know best is slowing changing and there is a recognition that we all have a role to play. \#9

In his study of integrated working Glasby (2004) noted hospital social workers are highly valued by their healthcare colleagues, however in a system under pressure and over stretched, tensions between the staff could quickly emerge. Complex, psychosocial assessments can be time-consuming and result in delays to the discharge process, which puts the working relationship under strain. Social workers need to be competent in their role and confident in their professional identify (Ambrose-Miller and Ashcroft, 2016) and be able to communicate this to their colleagues. Clarity of 
roles and responsibilities ensures optimum team working. These social workers stressed that a lack of understanding around their knowledge and expertise could negatively affect team working. A strong, positive inter-disciplinary relationship was dependent on mutual respect. Although many had good experiences of interdisciplinary working, some reflected on the challenges that it presented.

Sometimes our authority and expertise is questioned or challenged because it's not clear what our professional contribution is, what our skills are and what we add to the process. \#2

I think sometimes the others struggle to see us as equals as our particular skills are not well defined and we can become the bad guys who ask the hard questions and delay everything. \#12

There is unrelenting pressure to discharge people quicker and sicker. Sometimes there is obvious frustration about the work that we need to do. Sometimes it feels like once someone is deemed medically fit for discharge, everyone else just washes their hand of them. \#15

There was some support for Oliver's (2013) contention that working in interdisciplinary teams could lead to a loss of professional status and identity and could be viewed as a threat. Although, it was suggested that this much more likely if social workers did not have a strong sense of self-identity and were unable to clearly articulate their unique contribution.

\section{Ethical issues}

Social work by its very nature is fraught with conflicts, tensions, and ethical difficulties. (Banks, 2004, Cummings and Cockerham, 1997, Fantus et al 2017). The social workers in this study referred to ethical conflicts round the allocation and 
distribution of scarce resources, the increasing focus on managerialism and targets, inter-professional conflicts and the tensions between advocating for the patient and the responsibility the social worker had to their employing organization.

A number of the social workers acknowledged the difficulties around funding and the challenges of working within a climate of austerity but stressed that it was essential that they remained committed to their values. Whilst there was loyalty and commitment to employers, it was within the broader framework of professional values and ethics. There was however some concern that the scope for challenging practices, flexibility and interpretation was limited within the increasing focus on procedures and protocols. Research assessing moral distress amongst a diverse range of health care professionals found that for hospital social workers the highest indicators of moral distress were related to discharge planning rather than direct patient care (Houston et al 2013). Respondents in this study identified balancing the requirements of the employers with the needs of the patient as a source of ethical dilemmas and challenges.

You just feel the need to do your best for the patient and get them the best service that is possible, but there is always tension about who gets what when there are limited resources. You have to remember getting something for one person may mean its not there for another. \#18

I think you have to be clear what are you asking for and why. Yes there are rules but you can be creative and flexible rather than simply accepting. I'd say we are resourceful and aim to get the most of out what we have. \#20

For example these social workers operate within the Patient Choice Protocol developed by this Health and Social Care Trust. This policy states that patients must accept beds in a residential home within a 35 mile radius and this can present a 
dilemma for social workers. If a residential home placement is required but one cannot be secured close to home, patients do not have a right to wait for a place in a preferred location. The social worker aims to deliver the best outcome for the patient, but must also work within the relevant procedures and polices. A social work manager explained how this dilemma could be addressed,

Policies are there to guide and assist not to be implemented religiously. Social workers are not robots and must reflect on what is best in the long run. They will look at the context and use their knowledge to come to a decision. It is important to remember that we have professional judgement. \#21

There was a broad consensus that social workers had to find an appropriate balance between the needs of their clients and the expectations of employers. Flexibility and creativity were described as essential skills in this clinical context. Faced with austerity and shrinking resources the complexities of providing responsive needs-led care should not be underestimated. As a GP explained delivering services in deprived areas against a backdrop of austerity and cuts could be emotionally exhausting.

Don't underestimate the challenges of delivering services to the vulnerable in a context of cuts, targets, value for money and savings. Sometimes you feel like you are letting everyone down. It's not for the faint-hearted. \#30

\section{Improving service provision}

The participants in this study offered a number of suggestions to enhance the social work services in hospital. A key recommendation was the need for joint training and increasing opportunities for health and social care professionals to gain a better understanding of how their skills contributed to a whole systems approach. Differing ideologies between health and social care professionals had caused unnecessary conflict and friction. This was often frustrating and time-consuming and could have been avoided by more appropriate inter-disciplinary models of education. It was 
suggested that many professionals did not really understand the contribution of others and have been trained to focus only on their own professional goals. This was not conducive to joint working and in many ways seemed counter-intuitive. Previous research has concluded that providing training and development opportunities together are an obvious way to support staff members who will be working within an integrated team (Millar et al, 2016).

A nurse explained what she viewed as the contradictions in theory and practice.

I have never for the life of me, understood why we don't have some compulsory joint education or training. Delivering quality health and social care seems to be entirely based on multi-disciplinary working, yet if we are all part of a jigsaw shouldn't we at least have a basic understanding of how we all fit together. \#24

This view reiterated by an Occupational Therapist,

I really think we need to give more consideration to initiatives which encourage and facilitate a more joined up approach. We have an integrated system of health of social care but professionals are trained in silos. I think its particularly an issue for social workers, as we have a completely different ethos which to be honest is very poorly understood. We can all work to the same objectives if we had a better understanding of what we do and why. \#27

Taylor (1998) has acknowledged the need to balance individual needs and service needs in social work training. He suggested that initial social work training must focus on gaining an in-depth understanding the role and developing the essential knowledge and skills required to be a professional. The need for a much stronger emphasis on multiprofessional working is highlighted but he contends that this should be part in-service and post-qualifying training. Current health education models based largely on separate training for professionals may inadequately prepare students for collaboration and co- 
operation. There has been an acknowledgement within more recent policy documents and strategies that inter-disciplinary team working may require some blending of roles and flexibility to enable clinical demands to be met (DOH England, 2013, Bengoa, 2016). In 2016, a major review of health and social care in Northern Ireland placed a strong emphasis on primary care, augmenting general practice with multi-disciplinary teams including district nurses and health visitors. It noted "transformation in workforce requires a significant mind shift from a traditional silo approach to roles removing barriers that delineate one professional role from another” (Bengoa, 2016). Social workers reported concern that other professionals did not have a clear idea of the distinctive contribution of their work and as Baginsky (2014) has noted this may partly contribute to them being viewed in a negative manner. Social workers in interdisciplinary teams are often the only representative of their profession and this can result in marginalisation. In this study tensions occured when social workers were perceived by other team members to have spent too long on a discharge case and not exercised enough discretion or appeared to be adopting a risk averse approach. Social workers reported that occasionally it felt like a challenge to 'hold the line' on their professional values and identity.

Integration and collaboration should never mean assimilation. Social workers have a distinctive value base and will not compromise that because of pressure from other healthcare professionals. \#19

Participants repeatedly referred to the importance of good communication in collaborative working. There was almost universal agreement that effective communication skills should be considered as a key prerequisite for successful team working. The need for further training in communication specifically addressing the challenges of working as part of an interdisciplinary team was identified as a key issue for the social workers. A number of the participants suggested that some additional training on group dynamics, active listening and 
assertiveness would be particularly beneficial in this clinical context. Poor communication was identified as a significant impediment to effective team working.

I really think there is a need for further support and training on communication. Sometimes you can really struggle to be to get your point across in a team where you are coming from a different perspective. Assertiveness can come across as aggressiveness and that causes hostility and damages relationships. \#13

Perceived power imbalances on inter-disciplinary teams were thought by some to impact negatively on social workers ability to communicate effectively. There was a recognition that doctors and consultants were associated with power and control and these traditional hierarchical ideas could negatively impact on social workers ability to find and use their voices. As the complexity of patients' needs increase, there is a greater need for communication and negotiation with a greater number of people in their care pathway. Social workers need to communicate effectively in a team to ensure that patients are not discharged inappropriately into an unsafe environment (Green, 2007, Reisch, 2012).

\section{Conclusion}

This paper is based on an exploratory study based in one acute hospital in Northern Ireland it had a number of limitations. Time and financial constraints did not allow for interviews with service users or carers although it is hoped to include these in a larger follow up study. Also it is largely subjective and limited to the perceptions of those working in one geographical area. However despite these limitations, the findings offer some insights into hospital-based social work and provide evidence of how this role is experienced. 
The findings from this study illustrate the multi-dimensional nature of hospital-based social work and the breadth of expertise and skills. However, against a backdrop of austerity and a focus on reducing the length of hospital stays, discharge planning has become the dominant issue with less time available for the psychosocial aspects of health and illness. The emphasis on increasing hospital throughput has had major implications for these professionals, yet despite this, little research has been undertaken to assess their roles in this process and what factors enhance or impede their ability to work in this context.

Whilst there was a general agreement that this work was rewarding and fulfilling it could also be emotionally draining and poorly understood. Inter-professional working is increasingly becoming viewed as a central component in the delivery of healthcare and social workers make a unique contribution to these teams. The participants in this study reported that it involved both challenges and rewards. They highlighted the need for these changing and evolving roles to be reflected in education and training models. Inter-professional education, both formal and informal could be an effective means to strengthen teamwork and address some of the cultural, identity, power and communication issues affecting the dynamics of inter-professional teams health care teams. In order to ensure that social workers can continue to provide a holistic service it is essential that their unique role in inter-disciplinary teams is acknowledged, understood and supported. 


\section{References}

Abendstern, A., Hughes, J., Clackson, P., Sutcliffe, C and Challis, D. (2011) 'The pursuit of integration in the assessment of older people with health and social care needs', British Journal of Social Work. 41 (3) pp. 467-485.

Albrithen and Yalli, (2016) 'Peer interaction and hospital integration', International Social Work, 59 (1), pp. 129-140.

Ambrose-Miller, W. and Ashcroft R. (2016) 'Challenges faced by social workers as members of interprofessional collaborative health care teams', Health Social Work, 41 (2), pp. 101-110.

Baginsky, M. (2014) 'Social work in hiding? The views of other professionals on social workers and working with social workers'. Research, Policy and Planning, 30 (3), pp.143-154.

Banks, S. (2004) Ethics, Accountability and the Social Professions, Basingstoke, Palgrave Macmillan.

Bengoa, R (2016) Systems, Not Structures-Changing Health and Social Care, Belfast, DHSSPS. 
Barber, R.D. A.C. Kogan, A. Riffenburgh, and Enguidanos, S. (2015) 'A role for social workers in improving care setting transitions: A case study'. Social Work Health Care, 54 (3), pp.177-192.

Beder. J. (2006) Hospital Social Work: The Interface of Medicine and Caring: New York: Routledge, Taylor and Francis.

Beddoe, L (2013) 'Health social work: professional identity and knowledge', Qualitative Social Work, 12 (1), pp.24-40.

Bendor, S.J. ( 1998) The clinical challenge of hospital-based social work practice, Social Work in Health Care, 13 (2), pp.25-34.

Bull M. and Roberts J. (2001) 'Components of a proper hospital discharge for elders', Journal of Advanced Nursing, 35 (4), pp. 571-581.

Cornwell, J., Levenson, R., Sonola, L. and Poteliakhoff, E. (2012) Continuity of Care for Older Hospital Patients: A call for action, London, The King's Fund.

Cowles, L.A. and Lefcowitz, M.J. (1992) 'Interdisciplinary expectations of the medical social worker in the hospital setting', Health Social Work, 17 (1), pp. 57-65.

Cleak, H.M. and Turczynski, M. (2014) 'Hospital social work in Australia: Emerging trends or more of the same?', Social Work in Health Care, 53 (3), pp. 199-213. 
Craig, S. and Muskat, B. (2013) 'Bouncers, brokers and glue: The self-described roles of social workers in urban hospitals', Health and Social Work, 1 (1), pp. 7-16.

Cummings, S.M. and Cockerham, C. (1997) 'Ethical dilemmas in discharge planning for patients with Alzheimer's Disease', Health and Social Work, 22 (2), pp.101-108.

Davis, C. Milosevic, B. Baldry, E. and Walsh A. (2005) 'Defining the role of the hospital social worker in Australia: Part 2. A qualitative approach', International Social Work, 43 (3), pp. 289-299.

Department of Heath (England) (2013) Healthy Lives, Healthy People: A Public Health Workforce Strategy, DOH, London.

DHSSPS (2016) Health and Wellbeing 2026, Delivering Together, DHSSPS, Belfast.

Fantus, S., Greenberg, RA., Muskat, B. and Katz D. (2017) 'Exploring moral distress for hospital social workers', British Journal of Social Work, 47 (8) pp. 2273-2290.

Gerdes K.E, and Segal, E. (2011) 'Importance of empathy for social work practice: integrating new science', Social Work, 56 (2), pp.141-8.

Green, D. (2007). 'Risk and social work practice', Australian Social Work, 60 (4), pp.395-409. 
Glasby, J. (2007) Understanding Health and Social Care, Bristol, Policy Press.

Glasby, J. (2004) 'Discharging responsibilities? Delayed hospital discharges and the health and social care divide', Journal of Social Policy, 33 (4), pp.593-604.

Hall, P. 2005. 'Interprofessional teamwork: professional cultures as barriers', Journal of Inter-professional Care, 19 (1), pp.188-196.

Heenan, D and Birrell D. (2009) 'Organisational integration in health and social care: some reflections on the Northern Ireland experience', Journal of Integrated Care, 17 (5), pp.3-12.

Heenan D and Birrell D. (2018) The Integration of Health and Social Care in the UK: Policy and Practice. London Palgrave Macmillan.

Henwood, M. (2006) 'Effective partnership working: A case study of hospital discharge', Health and Social Care in the Community, 14 (5), pp. 400-407.

Houston, S., Casanova, M., Leveill, Schmidt, K., Barnes, S., Trungale, K. and Fine, R. (2013) The intensity and frequency of moral distress among different healthcare professionals'. AJOB Primary Research, 3 (2), pp.1-9.

Holliman, D.C., Dziegielewski, S.F. and Datta, P. (2001) 'Discharge planning and social work practice', Social Work in Health Care, 32 (3) pp.1-19. 
Hudson, B. (2002) 'Interprofessionality in health and social care. The Achilles heel of partnership?', Journal of Interprofessional Care, 16 (1), pp. 400-7.

Javadi M. and Zarea K. (2016) 'Understanding thematic analysis and its pitfall', Journal of Client Care, 1, (1), pp.33-39.

Judd R.G. and Sheffield S. (2010) 'Hospital social work: contemporary roles and professional activities', Social Work Health Care, 49 (9) pp.856-71.

Linn, C.J. and Tjung, J.J (2012) 'Discharge planning', International Journal of Gerontology, 6 (4), pp.237-240.

Majeed, M., Williams, D., Pollock, R., Amir, F., Liam, M., Foong, K. and Whitaker, C. (2012) 'Delay in discharge and its impact on unnecessary hospital bed occupancy', BMC Health Series Research, 12, pp. 410.

Millar, R., Brown, H. and Manjan, C. (2016) Integrated Care in Action, London, Jessica Kingsley.

McAlynn, M. and McLaughlin, J. (2008) 'Key factors impeding discharge planning in hospital social work', Social Work in Health Care, 46 (3), pp. 1-27.

McLaughlin, J. (2016) 'Social work in acute hospital settings in Northern Ireland: The views of service users, carers and multi-disciplinary professionals', Journal of Social Work, 16 (2), pp. 135-154. 
National Audit Office (2016) Discharging older patients from hospital, London, NAO.

NHS Providers (2015) Right Place, Right Time. Better Transfers of Care: A Call to Action. London, NHS Providers.

NHS England (2017) Delayed Transfers of Care www.england.nhs.uk/statistics

Northern Ireland Audit Office (2016) Managing Emergency Hospital Admissions November, Belfast, NIAO.

Oktay, J.S. Steinwachs, D.M, Mamon, J. L.R. Bone and Fahey, M. (1992) 'Evaluating social work discharge planning services for elderly people: access, complexity, and outcome', Health and Social Work, 1 (4), pp.290-298.

Oliver, C (2013) 'Social workers as boundary spanners: Reframing our professional identity for interprofessional practice', Social Work Education, 32 (6), pp. 773-784.

Oliver, D., Foot, C. and Humphries, R. (2014) Making our Health and Care Systems fit for an Ageing Population, London, The King's Fund.

Oliver, D. (2015) Improving Hospital Discharge and Intermediate Care for Older People, London, The King's Fund. 
Pethybridge, J (2004) 'How team working influences discharge planning from hospital: a study of four multi-disciplinary teams in an acute hospital in England', Journal of Interprofessional Care, 18 (1), pp. 29-41.

Reisch, M. (2012). 'The challenges of health care reform for hospital social work in the United States', Social Work in Health Care, 51, 873-893.

Swinkels, A. and Mitchel, T. (2009) 'Delayed transfer from hospital to community settings: the older person's perspective', Health and Social Care in the Community, 17 (1), pp. 45-51.

Taylor B.J. (1998) 'Service needs and individual qualifications: training Social Workers for the community care policy initiative and post-qualifying credits', Social Work Education, 17(1), pp.77-93. 
